\title{
Hybrid MMSE Precoding for Millimeter-Wave (mmW) Multi- user Massive MIMO Systems
}

\author{
Farah Raisa ${ }^{1}$, Khaizuran Abdullah ${ }^{2}$, Ahmad Fadzil Bin Ismail ${ }^{3}$, Asif Reza ${ }^{4}$, Hudah \\ Adibah Bt. Mohd. Ramli ${ }^{5}$ and Wahidah Hashim ${ }^{6}$ \\ 1,2,3,4,6 Department of Electrical and Computer Engineering, International Islamic \\ University Malaysia, Kuala Lumpur, Malaysia. \\ ${ }^{5}$ College of Computer Science and Info Tech, Universiti Tenaga Nasional \\ Malaysia \\ ${ }^{1}$ f.r.farah@ieee.org, ${ }^{2}$ khaizuran@iium.edu.my, ${ }^{3} a f \_i s m a i l @ i i u m . e d u . m y$, \\ ${ }^{4}$ asif.a.reza@ieee.org, ${ }^{5}$ hadibahmr@iium.edu.my, ${ }^{6}$ wahidah@uniten.edu.my
}

\begin{abstract}
Millimeter-wave (mmWave) cellular systems is considered to be the key enabling technology for the future $5 G$ wireless communication systems because of its high data rates, low latency, high system capacity, and huge available bandwidths. However, in order to meet the increasing demand, mmWave communications need to overcome certain challenges including high path loss and interference which can be reduced by applying large antenna arrays to achieve high beamforming gains. Although multi-user beamforming can improve spectral efficiencies, full digital beamforming strategies used in the conventional microwave systems increase the hardware cost and consumes high power for large number of antennas in mmW systems. In this paper, a multi-user hybrid precoding structure is proposed for mmWave massive-MIMO channels utilizing MMSE precoders at the BS with perfect channel knowledge. Simulations show that the sum-rate obtained by the proposed hybrid precoding scheme is nearly similar to the single-user rate and also performs better compared to other hybrid precoding approaches.
\end{abstract}

Keywords: Millimeter-wave; mmWave; Hybrid Precoding; MMSE

\section{Introduction}

In order to achieve high throughput, millimeter wave networks are one of the promising technology that can be used in future 5G wireless communication technology. The spectrum band which ranges from $30 \mathrm{GHz}$ to $300 \mathrm{GHz}$ is defined as the mm-waves frequencies [1]. As millimeter-Wave ( $\mathrm{mmW})$ spectrum has very small wavelength, so the available bandwidths are larger than the bandwidth those are used today. As a result, there is more available spectrum and a large number of small antennas can be used in this frequency band which will lead to greater system capacity. So, it is expected to play an important role for the future 5G wireless networks [2]. However, there are a number of challenges that mmWave is going to face and before making it widely used, it must be managed and solved in order to ensure maximum efficiency. Millimeter-Wave is subjected to severe attenuation and path loss due to noise and interference. So, these problems can be solved by antenna Beamforming and multi user MIMO.

Implementation of analog beamforming for mmWave systems using phase-shifters is popular due to low cost and simplicity of the structure [3-6]. The transceivers operating at high frequencies as in the case of millimeter wavebands support analog beamforming since the digital beamforming makes the system more complex with massive MIMO. Analog $\mathrm{BF}$ is considered as the major support particularly for indoor mm-wave environments adopting different beamforming training protocols [3-4]. To overcome the 
high path loss issue of the mm-wave frequencies, a large number of antennas are aligned and the RF chains are kept as minimum as possible to maintain simple circuit and low power consumption. The beam pointing angles are needed to aligned to face each other to form beam angle pair. However, these proposed methods were only applicable for singlestream transmission and also has large training overhead for large number of users. The performance of these low-power analog beamforming approaches, is very poor and not completely suitable for mm-wave channels. Fully digital beamforming architectures used in broadband microwave [7] is impractical for millimeter-wave systems due to high complicacy of the hardware and high consumption of power for many antennas.

The efficient beamforming architecture for mm-wave systems needs to be designed with both analog and digital processing [8-17]. Hybrid analog-digital beamforming involves dividing the MIMO processing into both analog and digital domains for multiuser data transmission with minimum interference. In order to maximize the average received SNR and thus the link budget, joint analog-digital beamforming algorithm was developed [9] with perfect CSI where the number of AFE chains were reduced with negligible performance loss to minimize the cost. Zero forcing (ZF) and phased zeroforcing (PZF) hybrid precoding scheme is proposed in several literatures [14] [15] performing ZF precoding at the $\mathrm{BB}$. The analog RF precoder is designed to obtain the large array gain and after that ZF precoding is carried out depending on the channel.

The hybrid BF algorithm for single-user mm-wave systems which assumes only limited knowledge of the channel [14] considers both the reciprocal and sparse environment. However, these proposed hybrid precoding algorithms do not utilize the characteristics of the mm-wave channel properly. As a result, when applied to $\mathrm{mmW}$ systems, these approaches will consume high power due to the use of high resolution $\mathrm{ADC}$ at the receiver. Efficient beamforming design for millimeter-wave channels considering the special characteristics of the channel is needed that can bring benefit in practical application for the massive number of antennas and which is compatible for millimetre-wave frequency bands. Table 1 shows the research works on hybrid precoding systems in mmWave networks.

Table 1. Comparison of Research Work by Others on Hybrid Beamforming

\begin{tabular}{|c|c|c|c|}
\hline $\begin{array}{c}\text { Author and } \\
\text { Year }\end{array}$ & Configuration & Advantages & Drawbacks \\
\hline Kim (2013) & $\begin{array}{c}\text { Joint baseband-RF } \\
\text { with perfect CSI }\end{array}$ & $\begin{array}{c}\text { Applicable for both single } \\
\text { and multi-user scenario }\end{array}$ & $\begin{array}{c}\text { High hardware } \\
\text { complexity }\end{array}$ \\
\hline $\begin{array}{c}\text { El Ayach } \\
(2013)\end{array}$ & $\begin{array}{c}\text { OMP-based } \\
\text { precoding with } \\
\text { partial channel } \\
\text { knowledge }\end{array}$ & $\begin{array}{c}\text { Low complexity precoding } \\
\text { technique with high } \\
\text { performance }\end{array}$ & Single-user Channel \\
\hline Han (2015) & $\begin{array}{c}\text { Optimal Hybrid } \\
\text { BF solution }\end{array}$ & $\begin{array}{c}\text { Achieves multi-user } \\
\text { capacity; }\end{array}$ & $\begin{array}{c}\text { mmWave channel } \\
\text { characteristics not } \\
\text { well discussed }\end{array}$ \\
\hline Huang & $\begin{array}{c}\text { Subarray } \\
\text { beamformer for } \\
\text { long range mmW }\end{array}$ & $\begin{array}{c}\text { Better system performance } \\
\text { for long range mmW } \\
\text { systems }\end{array}$ & $\begin{array}{c}\text { Single-user system is } \\
\text { discussed only. }\end{array}$ \\
\hline Khan (2012) & Hybrid BF for \\
MMB & $\begin{array}{c}\text { Provides Gb/s data rate for } \\
\text { long distance with high } \\
\text { mobility; Cell-edge } \\
\text { throughput performance } \\
10-100 \text { times better than } \\
\text { LTE-A }\end{array}$ & $\begin{array}{c}\text { Low efficiency and } \\
\text { high power } \\
\text { consumption }\end{array}$ \\
& & & \\
\hline
\end{tabular}




\begin{tabular}{|c|c|c|c|}
\hline Liang (2014) & $\begin{array}{l}\text { Phased Zero- } \\
\text { Forcing hybrid } \\
\text { precoding }\end{array}$ & $\begin{array}{l}\text { Low-complexity precoding } \\
\text { scheme approaches the } \\
\text { performance of the digital } \\
\text { ZF precoding }\end{array}$ & $\begin{array}{c}\text { Energy efficiency is } \\
\text { not discussed }\end{array}$ \\
\hline $\begin{array}{c}\text { Alkhateeb } \\
\text { (2013) }\end{array}$ & $\begin{array}{c}\text { Hybrid } \\
\text { beamforming with } \\
\text { partial channel } \\
\text { knowledge }\end{array}$ & $\begin{array}{l}\text { Utilizes the sparse nature } \\
\text { of the mmW channel; } \\
\text { better performance for } \\
\text { single user channel }\end{array}$ & $\begin{array}{l}\text { Only applicable for } \\
\text { single user }\end{array}$ \\
\hline $\begin{array}{l}\text { El Ayach } \\
(2014)\end{array}$ & $\begin{array}{l}\text { Sparse Precoding } \\
\text { for } \mathrm{mmW} \text { systems }\end{array}$ & $\begin{array}{l}\text { Low complexity precoding } \\
\text { solution utilizing the } \\
\text { sparse nature of the } \\
\text { mmWave channel; } \\
\text { Suitable for limited } \\
\text { feedback architecture. }\end{array}$ & $\begin{array}{l}\text { Optimization of } \\
\text { energy efficiency of } \\
\text { massive MIMO is not } \\
\text { discussed; Only } \\
\text { single user precoding } \\
\text { is considered }\end{array}$ \\
\hline $\begin{array}{l}\text { Zhang and } \\
\text { Huang } \\
\text { (2014) }\end{array}$ & $\begin{array}{l}\text { Joint Precoding of } \\
\text { baseband and RF }\end{array}$ & $\begin{array}{l}\text { Achieves same sum rate } \\
\text { compared to that of a } \\
\text { digital beamformer }\end{array}$ & $\begin{array}{c}\text { mmWave channel is } \\
\text { not considered }\end{array}$ \\
\hline Chen (2015) & $\begin{array}{l}\text { Hybrid Iterative } \\
\text { transceiver design }\end{array}$ & $\begin{array}{c}\text { Achieves same } \\
\text { performance as full } \\
\text { baseband precoding } \\
\text { scheme; Very small } \\
\text { number of RF chains used } \\
\text { to reduce complexity }\end{array}$ & $\begin{array}{c}\text { Not specially } \\
\text { designed for } \\
\text { mmWave systems; } \\
\text { higher complexity } \\
\text { and high energy } \\
\text { consumption }\end{array}$ \\
\hline
\end{tabular}

In this paper, an efficient hybrid precoding approach for $\mathrm{mmW}$ channel with comparatively low-complexity is proposed where precoding is done in both analog and digital domains for multi-user scenario. In this design, RF precoding is done to increase the transmit power and after that MMSE baseband precoding is adapted for multi-user system to mitigate the inter-user interference. The paper is arranged as follows: System model for the mmWave hybrid beamforming structure in discussed in Section 2. In Section 3, the proposed hybrid MMSE precoding design is presented and analysed under perfect CSI in Section 4. Results is illustrated in Section 5 and finally the paper is concluded in Section 6.

\section{System Model}

For the system model for mmWave propagation shown in Figure 1, it is considered that $N_{t}$ number of transmit antennas and $N_{R F}$ number of chains are connected at the BS. The base station communicates with the mobile stations of $K$ users, each of them equipped with $N_{r}$ number of receive antennas. 


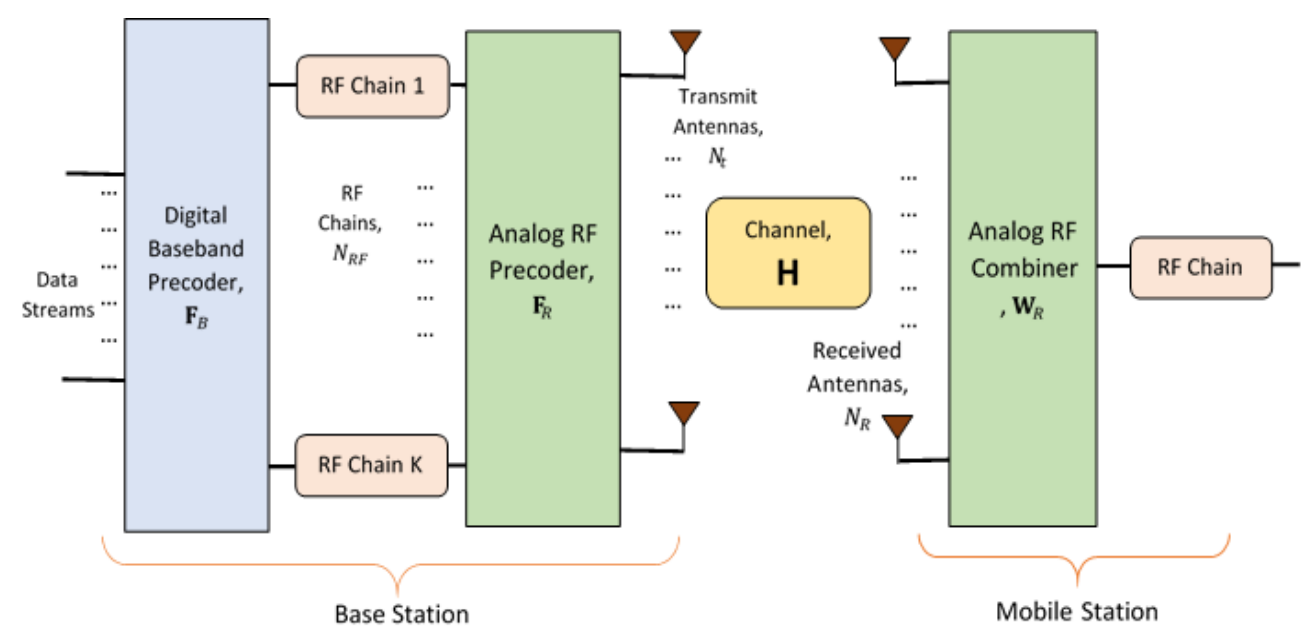

Figure 1. Block Diagram of mmWave Hybrid Beamforming Architecture

In multi-user beamforming system, each of the users supports single-stream transmission between the BS and the MS. So, the data streams are equal to total user $K$. It is also considered that the RF chains is same as the highest users being served at the same time i.e., the users are less than the RF chains. The transmitter at the BS executes $K \times K$ baseband precoder, $\mathrm{F}_{B}$, and $N_{t} \times K R F$ precoder, $\mathbf{F}_{R}$. The transmitted signal is then given by,

$$
x=\mathrm{F}_{R} \mathbf{F}_{B}
$$

In this equation, $s$ is the $K \times 1$ transmitted symbol vector such that, $\mathbb{E}\left[s s^{*}\right]=\frac{p}{K} I_{K}$, where, $P$ is the average transmit power. Analog phase shifters are being used to execute $\mathbf{F}_{R}$, so its entries are normalized in order to satisfy $\left|\left[\mathbf{F}_{R}\right]_{m, n}\right|^{2}=N_{t}^{-1}$. Moreover, each entry of $\mathbf{F}_{R_{1}}$ varies in phase-only and we normalize the magnitude so that it satisfies $\left[\mathrm{F}_{R}\right]_{m, n}=\frac{1}{\sqrt{N_{t}}} e^{j \phi_{m, n}}$, where $\phi_{m, n}$ is the quantized angle. The total power constraints at the BS is accomplished by normalizing $\mathbf{F}_{B}$ such that $\left\|\mathbf{F}_{R} \mathbf{F}_{B}\right\|_{F}^{2}=K$. A narrowband channel model is adopted [14] [17] where received signal observed by the $k^{\text {th }}$ user,

$$
r_{k}=\mathbf{H}_{k} \sum_{n=1}^{K} \mathbf{F}_{R} f_{n}^{B} s_{n}+n_{k}
$$

Where, $\mathbf{H}_{k}$ is the mm-wave channel matrix between BS and MS with dimension $N_{r} \times N_{t}, n_{k}$ is the additive Gaussian noise and $n_{k} \sim \mathcal{N}\left(0, \sigma^{2} I\right)$. The received signal at the MS of the $k^{\text {th }}$ user, where the combiner $w_{k}$ is used,

$$
y_{k}=w_{k}^{*} \mathbf{H}_{k} \sum_{n=1}^{K} \mathbf{F}_{R} f_{n}^{B} s_{n}+w_{k}^{*} n_{k}
$$

In the receiver's side, only analog RF beamforming is considered to simplify the architecture and lower the hardware cost.

\section{Channel Model}

The hybrid precoders $\left(\mathbf{F}_{R}, \mathbf{F}_{B}\right)$ and the hybrid combiners $\left(\mathbf{W}_{R}\right)$ are investigated depending on the channel matrix $\mathbf{H}$. In order to analysis the performance of the proposed hybrid precoding, a geometric mm-wave channel model is adopted with limited scattering characteristics for the channel, with each scatterer having a single propagation path. It is assumed that the channel is the sum of propagation paths, each having a scatterer and having $L_{K}$ number of scattering clusters for the channel $k$. Channel $\mathbf{H}_{k}$ can be expressed as 


$$
\mathbf{H}_{k}=\sqrt{\frac{N_{t} N_{r}}{L_{k}}} \sum_{l=1}^{L_{k}} \alpha_{k, l} a_{r}\left(\theta_{k, l}\right) a_{t}^{*}\left(\phi_{k, l}\right)
$$

where, $\theta_{k, l}$ is the angle of arrival and $\phi_{k, l}$ is the angle of departure of the $1^{\text {th }}$ path. The simulation of the proposed design is based on two types of antenna arrays, uniform planar arrays and uniform linear arrays. The array response vector for ULA antennas at the BS can be defined as

$$
\mathrm{a}_{t}\left(\phi_{k_{l} l}\right)=\frac{1}{\sqrt{N_{t}}}\left[1, \exp \left(j \frac{2 \pi}{\lambda} d \sin \left(\phi_{k_{l} l}\right)\right), \ldots, \exp \left(j\left(N_{t}-1\right) \frac{2 \pi}{\lambda} d \sin \left(\phi_{k_{l} l}\right)\right)\right]^{T}
$$

Where, $\lambda$ and $d$ are the wavelength and the distance between two antennas respectively.

\section{Hybrid MMSE Precoding for Multi-user MIMO}

Designing hybrid precoders and combiners at the BS and MSs efficiently to increase the total rate of the system is the main objective of this paper. The achievable rate for the user $K$ at the MS can be written as, after processing the received signal utilizing the combining vector,

$$
R_{k}=\log _{2}\left[1+\frac{\frac{P}{K}\left|w_{k}^{*} H_{k} f_{k}^{B}\right|^{2}}{\frac{P}{K} \Sigma_{m \neq \frac{R}{K}}\left|w_{k}^{*} H_{k} f_{k}^{B}\right|^{2}+\sigma^{2}}\right]
$$

Total sum-rate is given by,

$$
R t=\sum_{k=1}^{K} R_{k}
$$

There are only certain values for the analog precoding and combining vectors because only the quantized angles are available for the analog phase-shifters. These vectors are chosen from different beamforming codebooks such as general quantized BF codebooks, beamsteering codebooks and multi-resolution codebooks. As the analog beamsteering codebooks are previously used and shown to have better performance for single-user precoding schemes, in this paper these beamstearing codebooks are adopted for selecting the RF beamforming vectors. Here, for channels with single path, the beamforming vectors have similar type of array response vector which is represented by an angle. Let $\mathcal{F}$ and $W$ be the RF precoding vector codebook and RF combining codebook respectively.

Considering the sum-rate as the performance metric, $\mathbf{F}_{R}^{*},\left\{\mathrm{f}_{k}^{* \mathrm{~B}}\right\}_{k=1}^{K}$ and $\left\{\mathrm{w}_{k}^{*}\right\}_{k=1}^{K}$ are needed to obtained from the equation (4) to solve

$$
\begin{aligned}
& \left\{\mathbf{F}_{R}^{*},\left\{\mathrm{f}_{k}^{* \mathrm{~B}}\right\}_{k=1}^{K},\left\{\mathrm{w}_{k}^{*}\right\}_{k=1}^{K}\right\}=\arg \max \left(R_{\Omega}\right)
\end{aligned}
$$

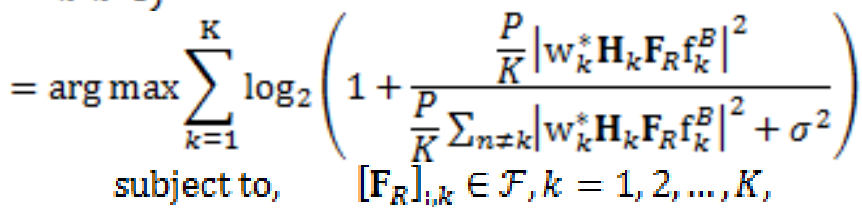

$$
\begin{aligned}
& \mathrm{w}_{k} \in W, k=1,2, \ldots, K \text {, } \\
& \left\|\mathrm{F}_{R}\left[\mathrm{f}_{1}^{B}, \mathrm{f}_{2}^{B}, \ldots, \mathrm{f}_{K}^{B}\right]\right\|_{F}^{2}=K .
\end{aligned}
$$

However, it is infeasible and unmanageable to obtain explicit solution of this maximization of the total sum-rate due to certain factors. The solution for the above equation needs to search for possible $\mathbf{F}_{R}$ and $\left\{w_{k}^{*}\right\}_{k=1}^{K}$ combinations over the $\mathcal{F}^{K} \times \mathcal{W}^{K}$. Moreover, the baseband precoder $\mathbf{F}_{B}$ is needed to incorporate with the RF beamforming vectors which needs the channel or effective channel feedback, resulting in large feedback and training overhead for this solution. There are several proposals for the solutions of the equation for designing the hybrid precoder and combiner. However, all these approaches 
are not suitable for the mm-wave systems. So, a modified algorithm is proposed for mmwave multi-user MIMO for many antenna elements.

\section{Modified Algorithm for Hybrid Precoding}

The proposed algorithm for the multi-user hybrid precoding is the modified and improved algorithm proposed in [14], where the total structure is divided into two stages. Firstly, the analog RF precoders and RF combiners are designed at the BS and MS respectively for single-user scenario avoiding the inter-user interference and the power of the signal per user is maximized without estimating the channel. The BF vectors for the $\mathrm{k}^{\text {th }}$ user are designed in such a manner so that at the signal power is highest at the $\mathrm{k}^{\text {th }}$ MS. The RF precoding scheme here is the typical analog precoding for single-user system that is used in other literatures [10] [12]. Efficient beam alignment algorithm is used which exploits the BF codebook to maximize the SNR. The hierarchical codebook utilizes a distance metric which can be optimized by using proper subarray methods.

After this stage, the digital BB precoder is constructed for multi-user MIMO and interuser interference in handled. Effective channels, $\bar{h}_{k}=\mathbf{w}_{k}^{*} \mathbf{H}_{k} \mathbf{F}_{R}, k=1,2, \ldots, K$. are trained by the base station with the mobile stations. A random vector quantization (RVQ) codebook $(\mathcal{H})$ is used to quantize these effective channels by their corresponding MS $(k)$, and then the channel vector index is send to BS with $B_{B}$ bits. Here, the MMSE (Minimum Mean Squared Error) precoding is then constructed in the BS depending on quantized channels.

\section{Spectral Efficiency Analysis with Perfect CSI}

In this section, the spectral efficiency is analysed which is achieved by the RF and baseband hybrid precoders. The performance is analysed for massive MIMO and with single-path channels.

Assuming that the effective channel knowledge is perfect and the RF beamsteering angles are of unending values. It is also assumed that all the codebooks including the RF codebooks for the for the BS and and MS and the RVQ codebook has the size of infinity.

It is considered that the channel is single-path so the scattering path $L_{k}=1$ for $k=1,2,3 \ldots K$.

The algorithm is used to construct the precoders and combiners with a few number of assumptions. The channel is considered single-path and the angle of RF precoding and combining vectors are continuous beamsteering vectors.

The effective channel can be written for $k$ user after constructing the analog precoders,

The effective channel matrix $\overline{\mathrm{H}}$ is given by,

$$
\begin{gathered}
\bar{h}_{k}=w_{k} \mathrm{H}_{k} \mathrm{~F}_{k} \\
=\sqrt{N_{t} N_{r}} \alpha_{k} a_{t}^{*}\left(\phi_{k}\right) \mathrm{F}_{R}
\end{gathered}
$$

$\overline{\mathrm{H}}=\mathrm{CA}_{t}^{*} A_{r}$

Where $C$ is the diagonal matrix with dimension $K \times K$ and $[C]_{k, k}=\sqrt{N_{t} N_{r}} \alpha$

The MMSE precoder of digital baseband depending on the effective channels can be expressed as, $\mathrm{F}_{B=} \overline{\mathrm{H}}^{\mathrm{H}}\left(\overline{\mathrm{H}^{\mathrm{H}} \mathrm{H}+\alpha \mathrm{I}}\right)^{-1}$ where $\alpha$ is the normalization factor and it is normalized to adjust elements according to the power constraints $\left\|\mathrm{F}_{\mathrm{R}} \mathrm{f}_{k}^{B}\right\|^{2}=1$.

The elements of the diagonal matrix $=\sqrt{\frac{N_{t} N_{r}}{\left(A_{t}^{*} A_{t}\right)_{k}^{-1}, k}}\left|\alpha_{k}\right|$

Then the achieved rate can be written as

$$
R_{k}=\log _{2}\left[1+\frac{S N R}{K}\left|\overline{\mathrm{h}}_{k}^{*} \mathrm{f}_{k}^{B}\right|^{2}\right]
$$




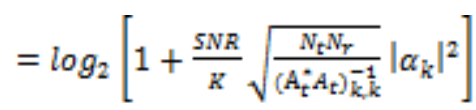

For single-user, the achievable rate is given by

$$
R_{\text {single }}=\log _{2}\left[1+\frac{S N R}{K} N_{t} N_{r}\left|\alpha_{k}\right|^{2}\right]
$$

As the precoders are designed in keeping with the algorithm with the assumptions stated before, the achievable rate of user $k$ and the single user achievable rate increases with the similar slope when SNR is high. The difference between these two types of achievable rates is always constant as it only depends on MSs and $N_{t}$, the number of BS antennas.

Virtual channel model is used to analyse the performance with large number of antennas which is only applicable for ULAs and UPAs. And the $N_{t}$ and $N_{r}$ are of finite numbers.

The virtual channel model has a finite number of $N_{t}$ and $N_{r}$ and fixed direction for the transmission and reception. Utilizing this channel model for the $\mathrm{k}^{\text {th }}$ user, the channel is given by

$$
\mathrm{H}_{k v}=\bar{A}_{Y} \mathrm{H}_{k}^{v} \bar{A}_{\mathrm{t}}^{*}
$$

Where, $\overline{A_{r}}$ and $\overline{A_{t}}$ are the virtual antenna array response vector for the MS and BS and $\mathrm{H}_{k}^{v}$ is the channel matrix for the $\mathrm{k}^{\text {th }}$ user.

The virtual channel model equation can be replaced in the equation (10) and can be rewritten as

$$
\mathrm{H}_{k v}=\sqrt{\frac{N_{t} N_{Y}}{L}} \sum_{m=1}^{N_{t} N_{r}} \gamma_{k, m} \mathrm{a}_{r}\left(\bar{\theta}_{k, m}\right) \mathrm{a}_{t}^{*}\left(\bar{\phi}_{k, m}\right)
$$

Here, $\bar{\phi}$ and $\bar{\theta}$ are the AoA and AoD for the virtual channel model.

The single-user rate is given by,

And the effective channel $\overline{\mathrm{H}}_{v}$,

$$
\mathrm{R}_{\text {single }}=\log _{2}\left(1+\frac{S N R}{K L} N_{t} N_{r}\left|\gamma_{k ; 1}\right|^{2}\right)
$$

$$
\overline{\mathrm{H}}_{v}=\mathrm{D}_{v} P_{v}
$$

$\mathrm{D}_{v}$ represents the diagonal matrix and $\mathrm{P}_{v}$ is the $K \times K$ matrix

Digital MMSE precoder,

$$
\mathrm{F}_{B}=\overline{\mathrm{H}}^{\mathrm{H}}\left(\overline{\mathrm{H}^{\mathrm{H}} \mathrm{H}}+\alpha \mathbf{I}\right)^{-1} \Lambda
$$

Where, $\alpha$ is the regularization factor and $\alpha=\frac{N_{r} \sigma_{n}^{n}}{\sigma_{s}^{2}}$, where $\sigma_{n}^{2}$ and $\sigma_{s}^{2}$ are noise variance and average transmit power respectively. So, the sum rate of user $K$ is given by

$$
\mathrm{R}_{K}=\log _{2}\left(1+\frac{S N R}{K}\left|\bar{h}_{k}^{*} f_{k}^{B}\right|^{2}\right)
$$

This equation represents the achievable rate for user $\mathrm{k}$ obtained by the proposed hybrid precoding structure. In the next section, the results of the simulation comparing the spectral efficiencies of the proposed design and other different beamforming approaches are presented.

\section{Simulation Results}

The performance of the proposed hybrid MMSE precoding approach is studied using numerical simulation and compared with other hybrid precoding designs of the literature discussed in the previous sections. The simulation is carried out in MATLAB simulator and in three different systems. MU-MIMO is considered in this analysis with $8 \times 8 \mathrm{BS}$ antennas and $4 \times 4 \mathrm{MS}$ antennas. Four mobile stations are considered and each having single-path transmission. The effective channel is assumed to be perfect with AoDs and AoAs to be uniformly distributed. The azimuths and elevations are distributed in $[0,2 \pi]$ and $[-\pi / 2, \pi / 2]$ respectively. Noise variance $\left(\sigma^{2}\right)$ is assumed to be 1 and SNR is $P \sigma^{2} / K$. The achievable rates of the proposed hybrid MMSE precoding technique is 
analyzed and compared with the single-user rate, analog beamforming and hybrid ZF precoding systems. The proposed hybrid precoding scheme performs very close to singleuser rate and ZF hybrid precoding and also achieves better gain over the analog beamforming approach. Figure 2(a) demonstrates the achievable sum-rate of different precoding approaches. For mmWave systems with a large number of BS antennas, the performance of the proposed scheme increases significantly. The simulation of the sum rate of the proposed hybrid precoding varying the BS antennas with fixed $N_{r}$ is shown in Figure 2(b). At $0 \mathrm{~dB}$ SNR, the sum rate of the proposed MMSE precoding scheme is 6 $\mathrm{bps} / \mathrm{Hz}$ whereas the sum rate of the $\mathrm{ZF}$ precoding scheme is $5.7 \mathrm{bps} / \mathrm{Hz}$. For lower SNR, the proposed scheme shows similar performance which is better than other hybrid precoding schemes.

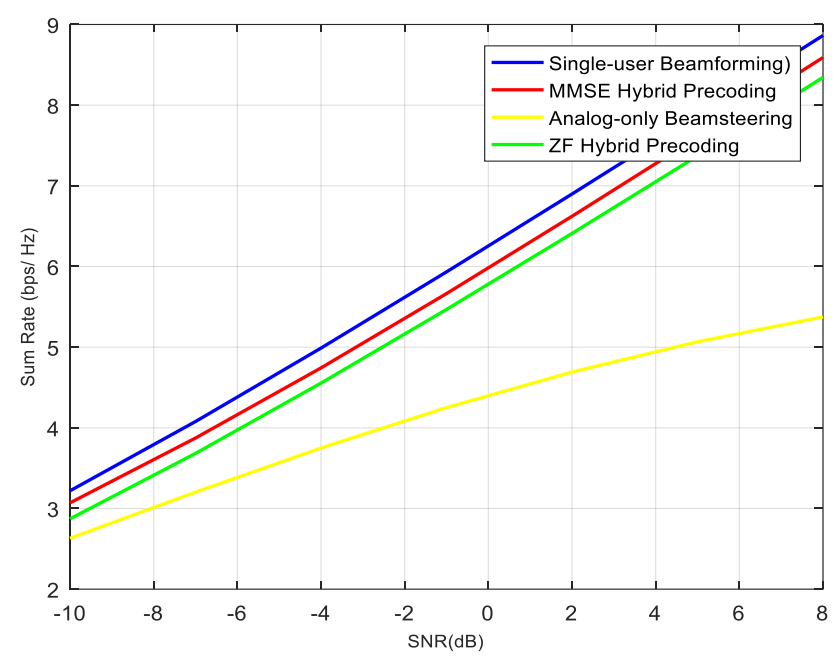

Figure 2 (a). Comparison of Different Schemes

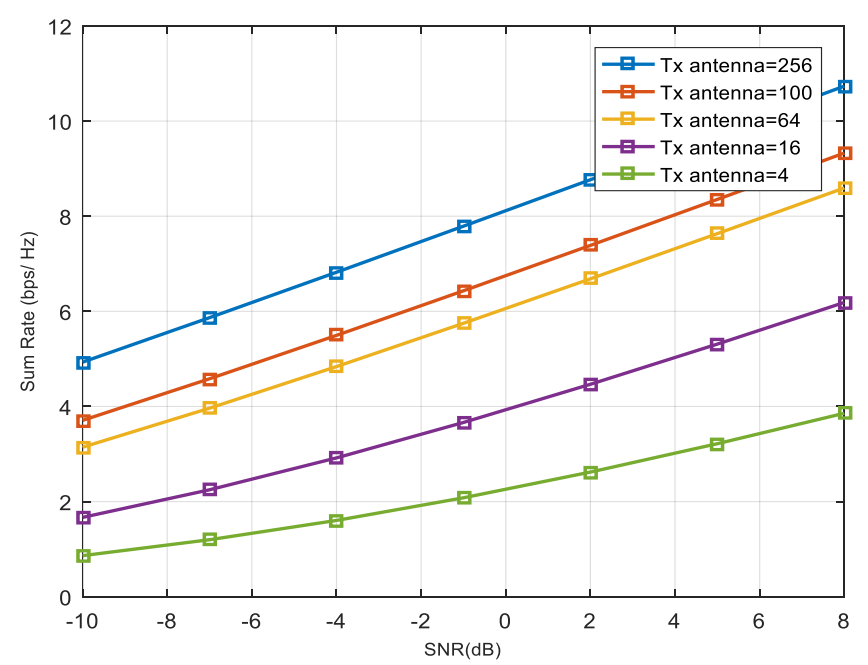

Figure 2 (b). Varying the Transmit Antennas

Figure 2. Results of Sum-rate Vs SNR curve for hybrid Precoding mmW Systems (a) Comparison of Different Schemes, (b) Varying the Transmit Antennas 


\section{Conclusion and Future Work}

In this paper, low-complexity hybrid precoding design is presented for mmWave multiuser communication with MMSE baseband precoding. Analyzing the proposed design under perfect channel, this approach has shown better performance compared to various precoding approaches in terms of achievable sum rate of the system. An extension of this research includes analyzing it under imperfect CSI which will be more practical approach for the future communication systems.

\section{References}

[1] Andrews, Jeffrey G., Stefano Buzzi, Wan Choi, Stephen V. Hanly, Angel Lozano, Anthony CK Soong, and Jianzhong Charlie Zhang. "What will 5G be?." IEEE Journal on Selected Areas in Communications 32, no. 6 (2014): 1065-1082.

[2] Akdeniz, Mustafa Riza, Yuanpeng Liu, Mathew K. Samimi, Shu Sun, Sundeep Rangan, Theodore S. Rappaport, and Elza Erkip. "Millimeter wave channel modeling and cellular capacity evaluation." IEEE journal on selected areas in communications 32, no. 6 (2014): 1164-1179.

[3] Tsang, Y. Ming, Ada SY Poon, and Sateesh Addepalli. "Coding the beams: Improving beamforming training in mmwave communication system." In Global Telecommunications Conference (GLOBECOM 2011), 2011 IEEE, pp. 1-6. IEEE, (2011).

[4] Hosoya, Ken'ichi, Narayan Prasad, Kishore Ramachandran, Naoyuki Orihashi, Shuya Kishimoto, Sampath Rangarajan, and Kenichi Maruhashi. "Multiple sector ID capture (MIDC): A novel beamforming technique for 60-GHz band multi-Gbps WLAN/PAN systems." IEEE Transactions on Antennas and Propagation 63, no. 1 (2015): 81-96.

[5] Hur, Sooyoung, Taejoon Kim, David J. Love, James V. Krogmeier, Timothy A. Thomas, and Amitava Ghosh. "Millimeter wave beamforming for wireless backhaul and access in small cell networks." IEEE Transactions on Communications 61, no. 10 (2013): 4391-4403.

[6] Gholam, Fouad, Javier Vía, and Ignacio Santamaría. "Beamforming design for simplified analog antenna combining architectures." IEEE Transactions on Vehicular Technology 60, no. 5 (2011): 23732378.

[7] Hampson, G. A., P. P. Roberts, M. Leach, A. J. Brown, T. Bateman, S. Neuhold, R. J. Beresford, "Microwave phased array digital beamforming system design challenges for SKA." In Microwave Conference (EuMC), 2015 European, pp. 710-713. IEEE, (2015).

[8] Heath, Robert W., Nuria Gonzalez-Prelcic, Sundeep Rangan, Wonil Roh, and Akbar M. Sayeed. "An overview of signal processing techniques for millimeter wave MIMO systems." IEEE Journal of Selected Topics in Signal Processing 10, no. 3 (2016): 436-453.

[9] Kim, Chanhong, Taeyoung Kim, and Ji-Yun Seol. "Multi-beam transmission diversity with hybrid beamforming for MIMO-OFDM systems." In 2013 IEEE Globecom Workshops (GC Wkshps), pp. 6165. IEEE, (2013).

[10] El Ayach, Omar, Robert W. Heath, Sridhar Rajagopal, and Zhouyue Pi. "Multimode precoding in millimeter wave MIMO transmitters with multiple antenna sub-arrays." In 2013 IEEE Global Communications Conference (GLOBECOM), pp. 3476-3480. IEEE, (2013).

[11] Han, Shuangfeng, I. Chih-Lin, Zhikun Xu, and Corbett Rowell. "Large-scale antenna systems with hybrid analog and digital beamforming for millimeter wave 5G." IEEE Communications Magazine 53, no. 1 (2015): 186-194.

[12] Huang, Xiaojing, Y. Jay Guo, and John D. Bunton. "A hybrid adaptive antenna array." IEEE Transactions on Wireless Communications 9, no. 5 (2010): 1770-1779.

[13] Alkhateeb, Ahmed, Omar El Ayach, Geert Leus, and Robert W. Heath. "Hybrid precoding for millimeter wave cellular systems with partial channel knowledge." In Information Theory and Applications Workshop (ITA) (2013) pp. 1-5. IEEE.

[14] Alkhateeb, Ahmed, Geert Leus, and Robert W. Heath. "Limited feedback hybrid precoding for multiuser millimeter wave systems." IEEE Transactions on Wireless Communications 14, no. 11 (2015): 6481-6494.

[15] Liang, Le, Wei Xu, and Xiaodai Dong. "Low-complexity hybrid precoding in massive multiuser MIMO systems." IEEE Wireless Communications Letters 3, no. 6 (2014): 653-656.

[16] Chen, Chiao-En. "An iterative hybrid transceiver design algorithm for millimeter wave MIMO systems." IEEE Wireless Communications Letters 4, no. 3 (2015): 285-288.

[17] El Ayach, Omar, Sridhar Rajagopal, Shadi Abu-Surra, Zhouyue Pi, and Robert W. Heath. "Spatially sparse precoding in millimeter wave MIMO systems." IEEE Transactions on Wireless Communications 13, no. 3 (2014): 1499-1513. 
International Journal of Future Generation Communication and Networking Vol.10, No.5 (2017) 